\title{
Wellbeing in the age of uncertainty
}

\section{Glenda H. Eoyang}

\section{Introduction}

Kia ora. I am pleased to be with you today. Thank you for the invitation to engage with your lively learning and action community. I am particularly happy to be exploring wellbeing with you at this time, in the middle of a global pandemic. In the time of COVID-19, I have come to think differently about health and wellbeing, and I am eager to share my insights and inquiries with you today.

I live in Minnesota now, in the Upper Midwest of the United States. If you follow the Mississippi River to its headwaters, you will be about 20 miles (32 kilometres) north of my home. I live with my sister and her daughter on the banks of a little lake. One of the gifts the pandemic brought me was being at home for the turn of four seasons. In pre-COVID-19 times I always seemed to be on a plane or in a different part of the world for the first snowfall, robin, tomato, or falling leaf. This year, I have delighted in them all!

I did not grow up here. I was born in Happy, Texas to a football-coaching father and an English-teaching mother. They moved my three sisters and me from one town to another as daddy's teams won (always a bigger school) or lost (a smaller one). Change was an 
ever-present part of life, as were the clouds and stars, but mountains were not.

Happy, and all the other towns we moved through, are on the Great Plains that stretch from Calgary in Canada to the Caprock south of Lubbock. That is more than 1,600 miles (2,600 kilometres). The most interesting thing about the Plains is that they are flat. I mean really flat. Most days, you can see 360 degrees of horizon. No hills or trees or tall buildings got in the way. I grew up accustomed to blowing wind and changing sky.

In that world of small towns and farmland, identity was easy. Methodist, Baptist, or Catholic; young or old; athlete or bookworm; long-time resident or transient were the cultural markers we saw and understood. Wellbeing was to find the comfort and stability that belonged to you and to settle into it for life. We were not aware of racial violence, corruption, or extreme poverty as part of our cultural equation, though surely our security put others at risk.

We were healthy when we avoided sickness. I remember a pink sugar cube that saved us from polio, Vicks VapoRub to cure a cough, and PeptoBismol to calm an upset tummy.

In the years since, I have come to think of wellbeing as something quite different, and I don't think that is just because I'm older. Chronic disease, environmental degradation, global markets, amazing medical technology, social and economic inequities, and mass communication have reframed how I think about health and wellbeing. I think of it now as "fit for function." A story will help illustrate.

A friend of mine, a physician in Canada, heard that an old friend was dying of cancer in Florida. He closed his practice and went to take care of her in her waning days. The way he tells the story is that he read her poetry, fed her smoothies, and rubbed her feet. She died, he said, the most well person he'd ever known. How can we understand such wellbeing? Even more importantly, how can we find it 
for ourselves, our families, and our communities in these uncertain times?

Since 1986 my colleagues at the Human Systems Dynamics Institute and I have explored the complex dynamics of social change. That inquiry has given us some ideas about how to find and hold wellbeing, in spite of circumstance. We are informed by state-ofthe-art science and wisdom of gifted practitioners. Our explorations include theory and practice, individual and community learning, and more questions than answers. I will share with you today in hopes that you will find hope and help. I also trust that you will critique and encourage and engage with us as our investigations continue.

\section{Complex adaptive systems}

Our emerging understanding of wellbeing derives from a paradigm of systems and change that emerged after the middle of the last century. Complexity science, chaos theory, and nonlinear dynamics provide scientific grounding for our work. Action research in government, business, personal transformation, and community development ground the practicality and application of the approach. On these foundations, we understand a complex adaptive system to be a collection of independent agents that interact with one another. The agents can be people, but they can also be cells, memes, political parties, institutions, or any other entity, in any medium. Together the agents contribute to a pattern of the whole system that is different from the sum of the parts. As the agents interact through sharing information, energy, money, physical connection, they create system-wide patterns. Those patterns, then, constrain the choices and actions of the agents in all future interactions. Such systems are called "self-organising," not because there is some psychological self involved. These systems are said to 
self-organise because future behavior is determined by other agents and interactions within the system, not by some external force or intention (Dooley, 1998).

Human systems are full of examples of complex adaptive, self-organising systems. Children on a playground, sports and project teams, families, neighbourhoods, activities in a logframe, and firms in an industry all demonstrate the power and potential of complex adaptive systems.

Change in such systems is unpredictable and impossible to control. It happens at multiple scales at the same time as parts self-organise within and between to move towards coherence at many levels simultaneously. It is open to dynamics of ever-larger and more unpredictable systems. It relies on nonlinear interactions, where cause and effect are impossible to separate. This kind of complex change in human systems is one reason why they are so hard to evaluate!

Wellbeing in a complex adaptive systems view is not a predetermined endpoint. It is an emergent pattern, resulting from multiple interactions of many different agents. It is continually changing and context sensitive. The goal of wellbeing is a level of dynamic equilibrium and coherence within and among systems at all scales of interaction. That is what wellbeing looks like in complexity-coherence and dynamic equilibrium. And, that is why my friend's friend could be healthy in her dying days.

\section{The role of tension}

In 20th century Western science, equilibrium was seen as a stopping point. A system roams around the problem space until it finds a resting place. It stops, and new sources of energy will be required to get it going again. Complex systems equilibrium is quite different. It is in perpetual motion. One change releases energy for another. A shift in one place or level sets conditions for unpredictable, uncontrollable 
change at another place. Sometimes the impact of change seems totally disconnected from its initiating shift, and sometimes the connection is stunning and immediate.

We understand this kind of change as the result of accumulation and release of tension. An avalanche provides a useful example of this kind of change. A mountain appears to be still because the forces and tensions within it are in a delicate balance. At the same time, within and around it, things are changing. A piece of gravel slides; a crystal of snow melts or freezes; the wind blows; and an animal burrows for food. Each of these tiny changes disrupts the surrounding structure, increasing the tension at boundaries nearby. At some critical point, the system can absorb no more tension. It becomes unstable, and any small change sends the system into cascading, system-wide, unpredictable, transformative change.

You see the same accumulation and release of tension at all scales of human interaction. Health turns into sickness when the immune system can no longer accommodate the viral load. A team breaks into conflict when irritation and frustration overcome norms and expectations. Divorce and falling in love both involve accumulation and release of tension over time. Economic collapse and violent extremism break through when the dynamic balance of tensions is disrupted. When my friend read poetry, made smoothies, and rubbed his friend's feet, the tensions of her terminal illness were released, and wellness emerged, even as she was dying.

These insights help you understand complex change and to recognise it in retrospect. For us in human systems dynamics, that is not enough. Our goal is to use the perception and understanding to inform action to influence the future, even when it cannot be controlled. 


\section{Using tensions to navigate wellbeing}

When you are aware of a tension in a system, you have three choices: Leave it alone and see what happens, shift conditions to increase the tension to move towards the breaking point, or change something to reduce tension and avoid an immediate collapse. The challenge is that dynamic tension management is not a "one and done" situation. Every time you take action to change one tension, you inadvertently affect many others. Releasing tension here can increase it there, increasing it here might also increase it there, leaving it alone may allow other forces to shift conditions and realign the whole system. When you acknowledge that change is accumulation and release of tension across a complex system, you recognise the need for constant surveillance and adaptive attention to see, understand, and influence whatever aspects of the system are within your scope of sight or understanding.

What does that mean for patterns of wellbeing for yourself, your family, and your community? It means you must be aware of and sensitive to differences that make a difference. You must be constantly vigilant to gauge the current levels of tension in the system and the risks and opportunities they allow. Finally, you use that intelligence to take an action and pay attention to the results. We call this process adaptive action, an iterative, inquiry process for agility in a complex system. It includes three questions.

\section{What?}

"What?" is the first question of adaptive action. It focuses on perception and data collection. What is happening now? What are the most relevant tensions in this moment? What is the current level of tension? What is holding the patterns in place? What are you not seeing? What assumptions or judgements influence your ability to see clearly? What might others be seeing? What scope and power do you have to act? 


\section{So what?}

"So what?" invites you into meaning making. So what is the most relevant tension? So what are your options? So what relationships exist or might be created? So what do others see or say? So what are the risks and benefits of various options? So what resources are available, including time and money? So what groups are possible allies or enemies? So what related experiences have you and others had? So what did you learn?

\section{Now what?}

"Now what?" moves you to action. Now what will you do? Now what will you say? Now what can you expect? Now what evidence will mark success? Now what is the urgency of action? Now what might be consequences that are not obvious?

Because of the nature of tensions and release, every "Now what?" shifts the balance of tensions in the system and creates a different dynamic equilibrium. When it does, you immediately shift into the next "What?" This cycle of inquiry, meaning making, and action continues for individuals and groups, as the system patterns shift and people adapt. It is in this adaptation that wellbeing emerges. It is more related to the capacity to see, understand, and influence than it is to any specific, stable outcome. Even when habitual, systemic racism and structures of privilege constrain the ability to see or influence, adaptive action can uncover surprising and potentially powerful options for action. Gifts of indigenous wisdom can bring particular grace and possibility into cycles of adaptive action. From this perspective, wellbeing is an active verb, not a static state that one moves towards or away from.

As we have worked with human systems at all scales, we have observed some common tensions that influence wellbeing. Each one of these tensions has the power to create or destroy a sense of 
wellbeing for individuals and groups. Structural racism, microaggressions, and explicit bias are patterns that emerge as such tensions. They destroy patterns of wellbeing. On the other hand, tensions of hope, generosity of spirit, and empathy have the opposite effect. I ask you to consider these tensions for yourself as you pursue your own adaptive action to create wellbeing. What patterns shape your behavior and expectations? Are they generative or destructive of wellbeing for you and your community of beings?

\section{Tensions for wellbeing}

In human systems dynamics, the most powerful tensions are held in what we call interdependent pairs. They are polarities that establish the tensions of the system's dynamic equilibrium. The two poles of the paradox are both desirable, but they are mutually exclusive. A healthy system is in an active, dynamical balance between the two extremes. It is this tension, and the ability to adapt it, that we think of as wellbeing in uncertainty. We will describe six interdependent pairs that we have found most useful as we seek wellbeing in this time of COVID-19 uncertainty.

\section{Stretch and fold}

All natural systems have a tendency to alternately expand and contract. Respiration in plants and animals is a great example. Expanding brings in new raw materials for life, and contraction expels waste. Learning follows the same dynamic pattern. Gather new insights and facts, then consolidate them with prior knowledge and skills. Friendships, projects, businesses, and diurnal cycles of work and rest all include both times of stretching and times of folding.

What does this have to do with wellbeing? Some people place a higher value on stretching. We have worked with health care professionals during COVID-19. They have been stretched physically, 
professionally, and emotionally as they respond to the unending demand. Some of them pine for a vacation in which they can fold and rest and consolidate their learning and mourning. Such a long time between stretch and fold can allow too much tension to accumulate or result in unexpected folds, when you least expect it.

When we coach people, we encourage them to find shorter, quicker opportunities to fold: Walking between patients, doing routine tasks, falling asleep, driving down the road, or on the path to the restroom. These "micro-breaks" allow them to manage the tension. They actively influence their dynamic equilibrium to harvest the energy of the tension without succumbing to its dangers.

\section{Stillness and motion}

In this interdependent pair, stillness isn't just the absence of motion. It is a conscious state of awareness without motion. You can experience it in this moment by focusing on your breath and inviting the rest of your physical, mental, and emotional body to come to rest. How does that feel? How quickly can you move from motion to stillness? That speed of transition is the key to wellbeing - not so much the amount of movement or stillness, but how quickly you can transition from one to the other.

Mindfulness, meditation, and many indigenous practices hone the capacity for stillness. In the Native American tradition, examples include vision quest and sweat lodge. Industrial cultures, on the other hand, value motion. In the context of, and in tension with stillness, motion can be of two types. It may be directed and intentional-motion towards a goal, or it may be random searching and wandering. Most of us have a bias towards directed movement, but in the human systems dynamics view, neither of these is better or worse than the other. The wellbeing challenge is to choose the kind of motion that will produce the results you want. If you have a specific 
thing to accomplish, focus and direction in motion are perfect. On the other hand, if you need to explore new territory, find an innovative approach, or test your own assumptions, then a random walk would serve you better.

The key to wellbeing is to:

1. see where you are in this continuum in a moment- "What?"

2. consider whether that is the most effective choice- "So what?"

3. choose some action to shift your current state to be most fit for function in a particular place and time.

\section{Memory and imagination}

History is an important factor in every complex adaptive system. The current state of the agents depends on everything and anything they have ever experienced. That is where memory comes in. It connects you, as an agent of the complex system, to the patterns that have emerged over time.

The future is equally important. Not because you can predict or control it. It is important because you can imagine what might be, and those possibilities inform which tensions you choose to address, and how you will address them.

The present moment — the moment in which you act—is informed both by your memory and your imagination. If you slide too far to memory, then you will be stuck in what was. If you slide too far into imagination, you will be distracted and dazzled by what might be. Either will shift you out of wellbeing and into dissatisfaction or despair. The key is to build capacity in both memory and imagination, and to engage in conscious adaptive action in each moment to bring the intelligence of both to support your action in the moment.

\section{Stable and unstable}

Nature provides us with two kinds of equilibrium. Stable equilibrium exists when the system returns to its original state after 
disruption. A pendulum hanging straight down is in stable equilibrium. Push it to one side, and it will eventually return to the same position. A pencil balanced on its point is a great example of unstable equilibrium. It is holding still, but if it is displaced, even a little bit, it will careen off into some new position rather than jumping back to where it started.

If you have ever watched a child learn to walk, you understand the powerful tensions between stability and instability. Without breaking symmetry, the child will never move. If they don't have the strength or balance to stabilise in motion, they will land on a diapered bottom. The ability to sense and respond in a moment is what the child learns in learning to walk. We need to learn the same thing as we move towards wellbeing.

All forms of human system engage in this same dance. Stability gives safety and security, it also damps the opportunity for creativity and innovation. Even when stability is a delusion, it can provide a short-term sense of safety. Instability, on the other hand, invites possibility in motion, but it brings the risk of the unexpected. These two-stability and instability_are woven together in the lives of people and their communities. In a state of wellbeing, you play with and within this tension, moving towards stability to consolidate, and towards instability to explore. Again, neither end of the pair is good or bad-in fact, you can think of them as competing goods. The question is where you should be in a given moment, and do you have the wisdom to know it and capacity to shift quickly to what is more useful in the moment.

\section{Zoom in and zoom out}

Complex adaptive systems function at multiple levels simultaneously. When self-similar patterns appear across multiple scales, the system has a special kind of complex coherence. Fractals are the most 
familiar example of such scale-free structuration. Biological systems and social ones, too, tend to reproduce the same tendencies and behaviours on micro-, meso-, and macroscopic scales.

Even when the repeating patterns are not so obvious in a complex system, different levels express a kind of functional coherence. Organelles form cells; cells form tissues; tissues shape organs; organs constitute systems; and systems inform the health of the whole. Each of these levels depends on those below and above. They influence and inform in both directions, too. This massive interdependency is sometimes misunderstood as hierarchy, but bottom-up influence is just as important as top-down effects.

When you consider your wellbeing as a state of complex adaptive systems, you recognise wellbeing at any level as indistinguishable from other levels. Your overall wellbeing is informed by both your neighbourhood and your ingrown toenail at the same time. When there is a dis-ease in the system, the cause may be at one level while the symptom is visible at another. To make the most of the multiple levels of cause and effect, you need to be able to see across them all. While it is impossible to see them all at the same time, it is possible to "zoom in and zoom out." When you recognise that wellbeing and its opposite depend on multiple levels of interacting dynamics, then you can shift from one to another to see what is happening ("What?"); understand it in useful ways ("So what?"); and take action to find a response that is most likely to be productive and generative ("Now what?")

\section{Expertise and inquiry}

The final tension that we see affecting wellbeing is an epistemological one. It deals with how and what we know. We will often ask people, "What do you know for sure? What do you wonder?" What they "know" may or may not be an ontological reality. The point that is 
most critical to wellbeing is how they stand in relation to what is real in the world. Their answers tell us a great deal about their current state of mind and options for moving towards greater wellbeing.

In a world of Newtonian causality, certainty, and predictability, expertise is highly valued. The person with the answer-especially the right answer-is the one with the power. In the uncertain world of complex adaptive systems, the opposite is true. No answer lasts for long, and the power goes to the person with the most useful question.

Like all the other interdependent pairs, both ends are true and useful. When the system is stable and predictable, answers are possible, and wellbeing depends on them. In the throes of chaotic transition, inquiry is the only way to sustain wellbeing for yourself and others.

This challenge is especially difficult for evaluators. Clients come to you for answers - at least they think they do. Sometimes you can deliver when you can ask your evaluation questions and come up with answers. Today, and in the increasingly complex future, your questions will fail to find answers. Really good questions will generate even more generative questions. That will be your job, to give answers when you can get them and to invite your clients into inquiry, when that is where wellbeing lies.

\section{Conclusion}

Wellbeing in these complex and uncertain times is not the absence of illness. It is not the same thing person to person. It isn't even the same thing for one person moment to moment. Instead, it is a dance of paradox: staying aware of emerging context and circumstance; tecognising differences that make a difference and tensions that hold generative potential; and making choices and taking action to hold and release tension. The goal is dynamic equilibrium and coherence, where the balance of tensions is forever in motion. 
I hope that this framing of complex adaptive systems, adaptive action, and a few key tensions will help you navigate wellbeing for yourself and your communities into our uncertain future.

\section{The author}

Glenda H. Eoyang, PhD

Executive Director

Human Systems Dynamics Institute

Email: geoyang@hsdinstitute.org 